\title{
Totally laparoscopic versus laparoscopic- assisted total gastrectomy for upper and middle gastric cancer: a single-unit experience of 253 cases with meta-analysis
}

\author{
Ke Chen, Yu Pan, Jia-Qin Cai, Di Wu, Jia-Fei Yan, Ding-Wei Chen, Hong-Mei Yu and Xian-Fa Wang*
}

\begin{abstract}
Background: Laparoscopic-assisted total gastrectomy (LATG) is the most commonly used methods of laparoscopic gastrectomy for upper and middle gastric cancer. However, totally laparoscopic total gastrectomy (TLTG) is unpopular because reconstruction is difficult, especially for the intracorporeal esophagojejunostomy. We adopted TLTG with various types of intracorporeal esophagojejunostomy. In this study, we compared LATG and TLTG to evaluate their outcomes.

Methods: From March 2006 to September 2015, 253 patients with upper and middle gastric cancer underwent laparoscopic total gastrectomy (LTG), 145 patients underwent LATG, and 108 patients underwent TLTG. The clinicopathological characteristics and postoperative outcomes were retrospectively compared between the two groups. Furthermore, a systematic review and meta-analysis were conducted.

Results: The operation time and estimated blood loss were similar between the groups. There were no significant differences in first flatus, diet initiation, and postoperative hospital stay. The surgical complication rates were $17.2 \%(25 / 145)$ and $13.9 \%(15 / 108)$ in the LATG and TLTG groups, respectively. The meta-analysis also revealed no significant differences in the operation time, estimated blood loss, time to first flatus, length of hospital stay, overall, and anastomosis-related complications among the groups.
\end{abstract}

Conclusions: TLTG is a feasible choice for gastric cancer patients, with comparable results to the LATG approach.

Keywords: Gastric cancer, Total gastrectomy, Intracorporeal anastomosis, Laparoscopy, Meta-analysis

\section{Background}

Gastric cancer is the fourth most common cancer worldwide and the second most frequent cancer-related cause of death in 2008 [1]. Surgery has been widely performed as the most effective treatment for resectable gastric cancer. Ever since it was first reported in 1994, the number of patients undergoing laparoscopic gastrectomy (LG) has been rapidly increasing. A randomized controlled trial has showed that laparoscopic gastrectomy is not inferior to open gastrectomy in patients with early distal gastric

\footnotetext{
*Correspondence: srrshwxf@163.com

Department of General Surgery, Sir Run Run Shaw Hospital, School of

Medicine, Zhejiang University, 3 East Qingchun Road, Hangzhou

310016Zhejiang Province, China
}

cancer. Large retrospective studies have also obtained acceptable oncologic outcomes [2]. In addition, laparoscopic surgery has the advantages of faster recovery, fewer complications, reduced hemorrhaging that reduces the likelihood of needing a blood transfusion, a smaller incision that reduces pain, the probability of intestinal obstruction, and the risk of wounding. Laparoscopicassisted gastrectomy (LAG) and totally laparoscopic gastrectomy (TLG) are two common methods of LG for gastric cancer. Usually, extracorporeal anastomosis with LAG was performed through a 5-7-cm small incision in the middle upper abdomen. However, extension of the laparotomy is often necessary to obtain a better view for secure anastomosis in obese

\section{) Bïomed Central}

(c) 2016 Chen et al. Open Access This article is distributed under the terms of the Creative Commons Attribution 4.0 International License (http://creativecommons.org/licenses/by/4.0/), which permits unrestricted use, distribution, and reproduction in any medium, provided you give appropriate credit to the original author(s) and the source, provide a link to the Creative Commons license, and indicate if changes were made. The Creative Commons Public Domain Dedication waiver (http://creativecommons.org/publicdomain/zero/1.0/) applies to the data made available in this article, unless otherwise stated. 
patients. Furthermore, the procedure is more difficult in cases with a short esophageal stump, because of the limited workspace even worse than larger laparotomy. TLG was established as a method for the intracorporeal resection and anastomosis using the laparoscopic technique. It has advantages over LAG, including a smaller wound and is less invasive [3-6].

Although the amount of totally laparoscopic distal gastrectomy (TLDG) performed for gastric cancer has gradually increased due to advancements in laparoscopic surgical instruments and the accumulation of operative experience, total laparoscopic total gastrectomy (TLTG) is not widely performed because of its technical difficulty, especially for the intracorporeal esophagojejunostomy.

Based on lots of laparoscopic experience from different laparoscopic operations, such as pancreatic and gastric surgery [7-13], we were encouraged to develop TLTG with different styles of interacorporeal esophagojejunostomy to treat middle and upper gastric cancer. This article compares the short-term achievements of patients who experience TLTG and laparoscopic-assisted total gastrectomy (LATG) in our center. A systematic review and meta-analysis were also conducted to further clarify the feasibility and safety of TLTG and to summarize the operative experience.

\section{Methods}

\section{Patients}

Between March 2006 and September 2015, 253 patients with middle or upper gastric carcinoma underwent laparoscopic total gastrectomy (LTG) at the Department of Gastrointestinal Surgery at the Sir Run Run Shaw Hospital, Affiliated Hospital of School of Medicine, Zhejiang University, China. The patients were divided into two groups according to reconstructive method, such as intracorporeal or extracorporeal reconstruction. All 253 patients were preoperatively examined by esophagogastroduodenoscopy (with biopsy), abdominal and pelvic computed tomography $(\mathrm{CT})$, chest radiography, electrocardiography, and basic blood testing. Endoscopic ultrasonography (EUS), liver magnetic resonance imaging (MRI), or chest CT was selectively performed as appropriate.

The patients' surgical characteristics (operative time, intraoperative hemorrhage), postoperative recovery (time to first flatus, time to initiate oral intake, complications, and length of postoperative hospital stay), and histopathologic indices (number of resected lymph nodes, surgical margins distance) were observed and compared between the two groups. Postoperative complications were classified as medical (cardiovascular, respiratory, or metabolic events; nonsurgical infections; deep venous thrombosis; and pulmonary embolism) or surgical (any anastomotic leakage or fistula, any complication that required reoperation, intra- abdominal collections, wound complications, bleeding events, pancreatitis, ileus, delayed gastric emptying, and anastomotic stricture). The Institutional Review Board of Sir Run Run Shaw Hospital of Zhejiang University approved this study protocol, and written informed consent was obtained from all patients before the investigation.

\section{Surgical procedure}

The patients underwent LTG with modified $\mathrm{D}_{2}$ lymph node dissection and Roux-en-Y reconstruction for gastric cancer. We previously performed LATG by using an anvil inserted via minilaparotomy. A policy of TLG was adopted at our hospital from its inception because we considered that it would confer several advantages. Therefore, we started performing TLTG using an anvil and intracorporeal purse-string suture technique in November 2007 and started using intracorporeal handsewn esophagojejunostomy in September 2012. Generally speaking, there are two approaches of intracorporeal esophagojejunostomy, including mechanical staplers and hand-sewn purse-string suture techniques. The details of the surgery are described in our previously published articles [14].

\section{Systematic review and meta-analysis}

We searched three major electronic databases (PubMed, EMBASE, and the Cochrane Library) for literature comparing LATG and TLTG published between January 1995 and September 2015. The following keywords were used: "laparoscopy," "laparoscopic," "gastric cancer," and "gastrectomy." The language of the articles was limited to English. Review articles, opinion pieces, and articles with no control group were excluded. Two investigators reviewed the titles and abstracts and assessed the full text to establish eligibility, and disagreements were resolved via discussion. The Newcastle-Ottawa Quality Assessment Scale was used for quality assessment of the observational studies. A threshold of six stars or above has been considered indicative of high quality.

\section{Statistical analysis}

All statistical analyses were performed using the Statistical Package for the Social Sciences $\left(\mathrm{SPSS}^{\circ}\right)$ version 16.0 (SPSS, Inc. Chicago, IL, USA). The differences in the measurement data were compared using the Student's $t$ test, and comparisons between groups were tested using a the $x^{2}$ test or the Fisher exact probability test. For the meta-analysis, Review Manage Version 5.1 (RevMan 5.1) software downloaded from Cochrane Library was used. Continuous variables were assessed using weighted mean difference (WMD), and dichotomous variables were analyzed using the risk ratio (RR). The anastomosis-related complications included anastomotic leakage, hemorrhage, and stricture or stenosis. To account for clinical 
heterogeneity, which refers to diversity in a sense that is relevant for clinical situations, we used the random-effects model based on DerSimonian and Laird methods. Potential publication bias was determined by conducting informal visual inspections of the funnel plots based on the complications. $P<0.05$ was considered to be statistically significant.

\section{Results}

\section{Baseline characteristics}

Table 1 summarizes the baseline characteristics of the two study groups. Of all 253 patients, 145 underwent LATG, while TLTG was performed on the other 108 patients. Both groups were well balanced for the variables (age, gender, BMI, comorbidity, ASA score, tumor size, tumor location, and TNM stage).

\section{Surgical outcomes in the LATG and TLTG groups}

Table 2 summarizes the operative outcomes and hospital courses of the LATG and TLTG groups. The operation time (234.8 $\pm 48.5 \mathrm{~min}$ versus $225.6 \pm 52.7 \mathrm{~min}, P=0.15$ ) was similar between the groups. However, the anastomotic times were lower in the LATG group than those in the TLTG group $(32.8 \pm 19.5 \mathrm{~min}$ versus. $47.5 \pm$ 23.2 min, $P<0.01)$. The mean blood loss was lower in the TLTG group than those in the LATG group but these differences were not significant $(137.6 \pm 54.7 \mathrm{~mL}$ versus. $125.3 \pm 62.8 \mathrm{~mL}, P=0.10$ ). The proximal margin

Table 1 Comparison of the clinicopathological characteristics

\begin{tabular}{|c|c|c|c|c|}
\hline & & $\begin{array}{l}\text { LATG } \\
(n=145)\end{array}$ & $\begin{array}{l}\text { TLTG } \\
(n=108)\end{array}$ & $P$ value \\
\hline Age (years) & & $57.3 \pm 12.5$ & $59.4 \pm 11.1$ & 0.18 \\
\hline \multirow[t]{2}{*}{ Gender } & Male & 98 & 73 & 0.99 \\
\hline & Female & 47 & 35 & \\
\hline $\begin{array}{l}\text { BMI index } \\
\left(\mathrm{kg} / \mathrm{m}^{2}\right)\end{array}$ & & $23.1 \pm 4.2$ & $23.5 \pm 3.5$ & 0.42 \\
\hline \multirow[t]{2}{*}{ Comorbidity } & Absence & 97 & 76 & 0.56 \\
\hline & Presence & 48 & 32 & \\
\hline \multirow[t]{3}{*}{ ASA classification } & I & 80 & 65 & 0.60 \\
\hline & $\|$ & 58 & 37 & \\
\hline & III & 7 & 6 & \\
\hline Tumor size $(\mathrm{cm})$ & & $4.3 \pm 2.0$ & $4.0 \pm 1.8$ & 0.23 \\
\hline \multirow[t]{2}{*}{ Tumor location } & Middle & 36 & 33 & 0.31 \\
\hline & Upper & 109 & 75 & \\
\hline \multirow[t]{2}{*}{ Histology } & Differentiated & 84 & 67 & 0.51 \\
\hline & Undifferentiated & 61 & 41 & \\
\hline \multirow[t]{3}{*}{ TNM stage } & $\mathrm{IA} / \mathrm{IB}$ & $54 / 28$ & $28 / 25$ & 0.45 \\
\hline & $\|A /\| B$ & $18 / 9$ & $14 / 13$ & \\
\hline & $\|\mathrm{A} /\| \mathrm{I} \mathrm{B} / \| \mathrm{I}$ & $12 / 10 / 14$ & $13 / 7 / 8$ & \\
\hline
\end{tabular}

distance and number of retrieved lymph nodes were not significantly different between the two groups. Postoperative outcomes include the first flatus time (3.4 \pm 1.0 days in the LATG versus $3.4 \pm 1.1$ days in the TLTG, $P=$ $0.19)$, diet start time ( $4.5 \pm 1.3$ days versus $4.4 \pm 1.4$ days, $P=0.56)$, and duration of postoperative hospital stay ( $9.4 \pm 2.5$ days versus $9.2 \pm 3.0$ days, $P=0.56$ ).

Table 3 shows the postoperative complications in the two groups. The postoperative complications are listed in Table 3. There was no in-hospital mortality and 30day mortality. Complications developed in $17.2 \%(25 /$ 145) of patients in the LATG group and $13.9 \%(15 / 108)$ of patients in the TLTG group. There were no significant differences in overall postoperative complications, surgical complications, or medical complications between the two groups.

\section{Outcomes of a systematic review and meta-analysis}

A total of 86 articles were identified in the three major electronic databases using the aforementioned search strategy. The titles and abstracts were reviewed, and articles without comparison of LATG and TLTG were excluded. Only three articles remained [15-17]. Including the present data, there were 816 participants in three studies (237 patients in the LATG group and 355 patients in the TLTG group). According to the NewcastleOttawa Quality Assessment Scale, all three studies received eight stars. The characteristics and methodological quality assessment scores of the included studies are shown in Table 4.

The results of meta-analysis are summarized in Table 5 . Meta-analysis of the operation time (WMD $=11.72 \mathrm{~min}$, $95 \%$ confidence interval $(\mathrm{CI})-2-94$ to $26.38, P=0.12$ ) (Fig. 1a) and anastomotic time showed no significant difference between the two groups (WMD $=-5.36 \mathrm{~min}$, $95 \% \mathrm{CI}-23.29$ to $12.57, P=0.56$ ) (Fig. 1b). There was also no significant intraoperative blood loss difference between the two groups (WMD $=80.39 \mathrm{ml}, 95 \% \mathrm{CI}$ -77.33 to $238.12, P=0.32$ ) (Fig. 1c).

The length of proximal resection margin was similar for both groups. However, the number of harvested lymph nodes of TLTG was more than that of LATG with a marginal difference $(\mathrm{WMD}=-2.11 \mathrm{~cm} ; 95 \% \mathrm{CI}-4.28$ to $0.06, P=0.06$ ) (Fig. 1d).

With regard to postoperative recovery outcomes, such as time to flatus and oral intake and duration of postoperative hospital stay, all outcomes showed no significant difference between the two groups (Fig. 2). Besides, none of the included studies reported mortality, and the overall and anastomosis-related complications were similar between the groups (Fig. 3). Visual inspection of the funnel plot of the anastomosis-related complications revealed symmetry, indicating no serious publication bias (Fig. 4). 
Table 2 Comparison of surgical outcomes and postoperative recovery

\begin{tabular}{llll}
\hline & LATG $(n=145)$ & TLTG $(n=108)$ & $P$ value \\
\hline Operation time (min) & $234.8 \pm 48.5$ & $225.6 \pm 52.7$ & 0.15 \\
Anastomotic time (min) & $32.8 \pm 19.5$ & $47.5 \pm 23.2$ & $<0.01$ \\
Estimated blood loss (mL) & $137.6 \pm 54.7$ & $125.3 \pm 62.8$ & 0.10 \\
Harvested lymph nodes & $31.2 \pm 10.4$ & $32.8 \pm 8.9$ & 0.20 \\
Proximal resection margin (cm) & $4.3 \pm 1.7$ & $4.6 \pm 1.6$ & 0.16 \\
First flatus (days) & $3.4 \pm 1.0$ & $3.4 \pm 1.1$ & 0.19 \\
Diet start time (days) & $4.5 \pm 1.3$ & $4.4 \pm 1.4$ & 0.56 \\
Postoperative hospital stay (days) & $9.4 \pm 2.5$ & $9.2 \pm 3.0$ & 0.56 \\
\hline
\end{tabular}

\section{Discussion}

Although laparoscopic surgery is frequently performed for the treatment of gastric cancer and LATG has been a very common approach, TLTG is not widely performed because of its technical difficulty. Esophagojejunal anastomosis after TLTG is one of the difficult procedures because of the difficulties in purse-string suturing and anvil placement. Moreover, concerns about the potential for high morbidity and mortality rates limited the enthusiasm for intracorporeal esophagojejunostomy. With recent advancements of laparoscopic instruments and the accumulation of operative experience, esophagojejunostomy can now be completed laparoscopically. In this study, we compared the results of TLTG to LATG in contemporary patient cohorts at a single institution over the same time period with the diagnosis of a gastric cancer. We included our initial experience with TLTG and found that outcomes were still quite comparable to the LATG approach.

Table 3 Comparison of postoperative complications

\begin{tabular}{llll}
\hline & LATG $(n=145)$ & TLTG $(n=108)$ & $P$ value \\
\hline Total complication & 25 & 15 & 0.42 \\
Surgical complications & 20 & 13 & 0.63 \\
Anastomotic leakage & 1 & 1 & \\
Anastomotic stricture & 2 & 3 & \\
Intracorporeal hemorrhage & 1 & 2 & \\
Abdominal abscess & 4 & 1 & \\
Stasis & 3 & 2 & \\
Pancreatic leakage & 2 & 1 & 0.43 \\
Ileus & 3 & 1 & \\
Lymphorrhea & 1 & 1 & \\
Wound infection & 3 & 1 & \\
Medical complications & 5 & 2 & \\
Pulmonary embolism & 0 & 1 & \\
Pulmonary infection & 4 & 0 & \\
Deep venous thrombosis & 1 & & \\
\hline
\end{tabular}

The safety of operation is an important focus for surgeons who perform TLTG. In our research, only 15 patients $(13.9 \%)$ suffered from postoperative complications. Both the results of meta-analysis and our data showed that the similar complications occurred in the two groups. Based on the further analysis of anastomosisrelated complications, such as stenosis, anastomotic leakage, and hemorrhage, among 108 patients, only 4 patients suffered from complications that were directly connected with anastomosis in the group of TLTG. Therefore, the rate of the complications related to anastomosis was $3.7 \%$ with no significant difference by data of our center or meta-analysis.

Anastomotic leakage was one of the most frequent reconstruction-related complications and occurred in $1.1 \%$, which is comparable to the ratio in open surgery. The leaks, followed by intra-abdominal abscess, required no intervention. Linear stapler side-to-side esophagojejunostomy could reduce anastomotic stenosis, because a stoma larger than 30-mm diameter can be created, when $45-\mathrm{mm}$ staplers are used [18]. This is one of the advantages of the linear-stapled method over circular stapling with regard to reducing anastomotic stricture. The reduced risk of anastomotic stenosis could contribute to a better quality of life for patients, because the symptoms of stenosis are one of the most important factors impairing the quality of life in patients after gastrectomy. However, the esophagojejunostomy made by linear or circular stapler could increase the risk of anastomotic hemorrhage. In our center, we started using intracorporeal hand-sewn esophagojejunostomy in September 2012, and there were no anastomotic hemorrhages after that.

Because the reconstruction portion of TLTG can be difficult, some researchers believe that longer operation time adversely affects patient outcome. In our current study, the operation time for TLTG was not longer than LATG. On the basis of our TLTG experience, two points contribute to this: First, the procedure of anastomosis was simplified using modified intracorporeal esophagojejunostomy techniques. Second, the opening and closure of minilaparotomy are exempted in the TLTG, which 
Table 4 Characteristics of included studies

\begin{tabular}{|c|c|c|c|c|c|c|c|c|}
\hline \multirow[t]{2}{*}{ Author } & \multirow[t]{2}{*}{ Nation } & \multirow{2}{*}{$\begin{array}{l}\text { Study } \\
\text { type }\end{array}$} & \multirow{2}{*}{$\begin{array}{l}\text { Publication } \\
\text { year }\end{array}$} & \multirow{2}{*}{$\begin{array}{l}\text { Study } \\
\text { period }\end{array}$} & \multicolumn{2}{|c|}{ Sample size } & \multirow{2}{*}{$\begin{array}{l}\text { Quality } \\
\text { scores }\end{array}$} & \multirow[t]{2}{*}{ Details of IE } \\
\hline & & & & & $\overline{\text { LATG }}$ & $\overline{\text { TLTG }}$ & & \\
\hline Kim & Korea & Pros & 2013 & 2010-2011 & 23 & 90 & 6 & Functional end-to-end \\
\hline Jung & Korea & Retro & 2013 & 2004-2012 & 47 & 40 & 6 & OrVil ${ }^{\mathrm{TM}}$ \\
\hline Ito & Japan & Pros & 2014 & $2001-2012$ & 46 & 117 & 6 & OrVil ${ }^{\mathrm{TM}}$ \\
\hline
\end{tabular}

Retro retrospective observational study, Pros prospective observational study, IE intracorporeal esophagojejunostomy

can shorten the operation time by 15 min. Certainly, the learning curve also effects on the operation time. What is more, skillful surgeons are capable of performing the operation safer and faster than unskilled surgeons.

Compared with the incision at the epigastrium required by LATG, the incision in TLTG is smaller. Therefore, TLTG is the better cosmetic solution. However, it is not clear whether TLTG is really less invasive than LATG or provides many clinical benefits to the patient in addition to cosmetic factors. Based on our data, the intraoperative blood loss in the group of TLTG was less than that in the group of LATG, but this value was not statistically significant. In the group of LATG, blood loss might increase due to skin incision and anastomosis through small skin incision by hand manipulation. Moreover, the esophageal stump must be pulled out from the abdominal cavity when LATG is conducted. The pulling puts great pressure on the esophageal stump and might even cause tearing and bleeding of the spleen envelope. However, this result should be interpreted prudently for the variation in blood loss between studies was high, with heterogeneity as a result of different methods of estimating blood loss.

Oncological results critically measure the success of laparoscopic surgery of malignant tumors. With short follow-up times, the main indicators of oncological quality are numbers of retrieved lymph nodes and surgical resection margin. It is our opinion that a technically similar oncologic resection can be performed regardless of whether the LATG or TLTG approach is used. As such, we would argue that neither procedure is technically superior nor that harvesting an adequate number of lymph nodes is largely dependent on the technique of the surgeon and on pathologic analytic variability. However, the meta-analysis demonstrated that the number of harvested lymph nodes of TLTG was more than that of LATG with a marginal difference $(P=0.06)$. However, in the included studies and our center, surgeons perform LATG during their early period and TLTG during the late period. The amount of dissected laparoscopic lymph nodes closely connects with the surgical skills. And thus, such time difference seemed to connect to the clinically obvious differences in the resection of lymph nodes.

In recent years, various modified intracorporeal esophagojejunostomy techniques have been reported, such as laparoscopic purse-string suture technique using Endo Stitch (Covidien) [19], Endo-PSI (Hope Electronics) [20], or a hemi-double stapling technique [21]. Another two intracorporeal reconstruction methods may be most representative; one using a transorally inserted anvil (OrVil; Covidien) to make an end-to-side esophagojejunostomy [22], the other using linear staplers to make a side-to-side anastomosis [23]. However, the optimal method for esophagojejunostomy in LTG remains to be established. The OrVil method carries the possible risk of pharyngeal or esophageal injury resulting from the passage of the anvil head at the level of the tracheal bifurcation. This can

Table 5 Pooled short-term outcomes of meta-analysis

\begin{tabular}{|c|c|c|c|c|c|c|c|}
\hline \multirow[t]{2}{*}{ Outcomes } & \multirow{2}{*}{$\begin{array}{l}\text { Number of } \\
\text { studies }\end{array}$} & \multicolumn{2}{|c|}{ Sample size } & \multirow{2}{*}{$\begin{array}{l}\text { Heterogeneity } \\
\left(P, P^{2}\right)\end{array}$} & \multirow{2}{*}{$\begin{array}{l}\text { Overall } \\
\text { effect size }\end{array}$} & \multirow{2}{*}{$\begin{array}{l}95 \% \mathrm{Cl} \\
\text { of overall } \\
\text { effect }\end{array}$} & \multirow{2}{*}{$\begin{array}{l}P \\
\text { value }\end{array}$} \\
\hline & & LATG & TLTG & & & & \\
\hline Operation time (min) & 4 & 261 & 355 & $0.06,59 \%$ & WMD $=11.72$ & $-2.94 \sim 26.38$ & 0.12 \\
\hline Anastomotic time (min) & 2 & 192 & 148 & $<0.01,98 \%$ & $\mathrm{WMD}=-5.36$ & $-23.29 \sim 12.57$ & 0.56 \\
\hline Blood loss (ml) & 2 & 191 & 225 & $0.02,83 \%$ & WMD = 80.39 & $-77.33 \sim 238.12$ & 0.32 \\
\hline Harvested lymph nodes & 3 & 215 & 238 & $0.60,0 \%$ & $W M D=-2.11$ & $-4.28 \sim 0.06$ & 0.06 \\
\hline Proximal margin $(\mathrm{cm})$ & 3 & 215 & 238 & $0.26,26 \%$ & WMD $=-0.06$ & $-0.37 \sim 0.26$ & 0.73 \\
\hline First flatus (days) & 3 & 215 & 238 & $0.44,0 \%$ & $W M D=-0.01$ & $-0.19 \sim 0.16$ & 0.88 \\
\hline Diet start time (days) & 3 & 215 & 238 & $0.12,53 \%$ & $W M D=0.37$ & $-0.15 \sim 0.90$ & 0.17 \\
\hline Hospital stay (days) & 3 & 215 & 238 & $0.63,0 \%$ & $W M D=0.32$ & $-0.31 \sim 0.96$ & 0.32 \\
\hline Overall complications & 2 & 168 & 198 & $0.71,0 \%$ & $\mathrm{RR}=1.31$ & $0.78 \sim 2.20$ & 0.30 \\
\hline Anastomosis-related complications & 4 & 261 & 355 & $0.46,0 \%$ & $R R=1.26$ & $0.60 \sim 2.65$ & 0.55 \\
\hline
\end{tabular}




\section{A LATG TLTG Mean Difference \\ Stuckvor Subgroup Mean SD Total Mean SD Total Weight N, Random, 95\% Cl Yea \\ Kim $\quad \begin{array}{llllllllll} & 158.5 & 45.5 & 23 & 166.4 & 47.5 & 90 & 23.2 \% & -7.90[-28.93,13.13] & 2013\end{array}$ \\ Jung $\quad \begin{array}{lllllllll}261.5 & 77.3 & 47 & 220.2 & 65.2 & 40 & 15.5 \% & 41.30[11.36,71.24] & 2013\end{array}$ \\ $\begin{array}{lrrrrrrrrr}\text { Ito } & 257.5 & 50 & 46 & 243 & 46.5 & 117 & 28.1 \% & 14.50[-2.23,31.23] & 2014 \\ \text { The present study } & 234.8 & 48.5 & 145 & 225.6 & 52.7 & 108 & 33.2 \% & 9.20[-3.49,21.89] & 2015\end{array}$ \\ Total $(95 \% \mathrm{Cl}) \quad 261 \quad 355 \quad 100.0 \% \quad 11.72[-2.94,26.38]$ Heterogeneity: Tau $^{2}=126.53 ; \mathrm{Chi}^{2}=7.27, \mathrm{df}=3(\mathrm{P}=0.06) ; \mathrm{I}^{2}=59 \%$ \\ Test for overall effect: $Z=1.57(P=0.12)$}



B
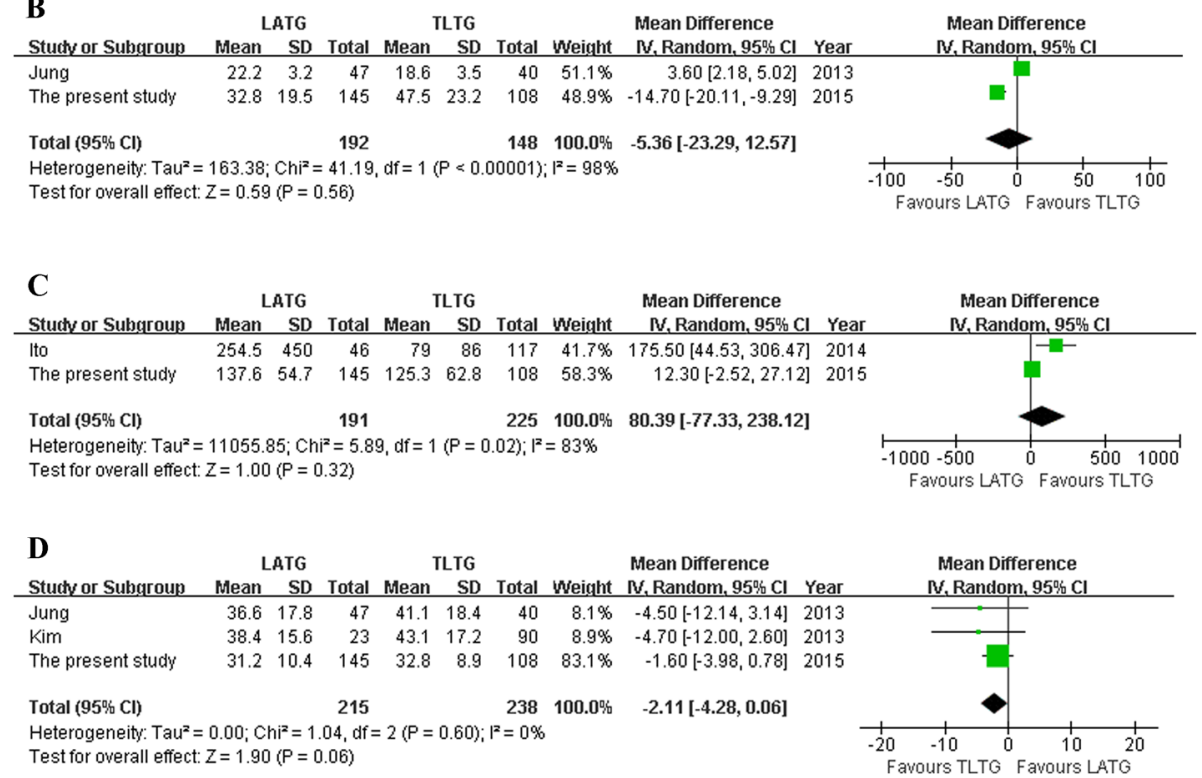

Fig. 1 Meta-analysis of the pooled data. a Operation time. b Anastomotic time. c Blood loss. $\mathbf{d}$ Harvested lymph nodes

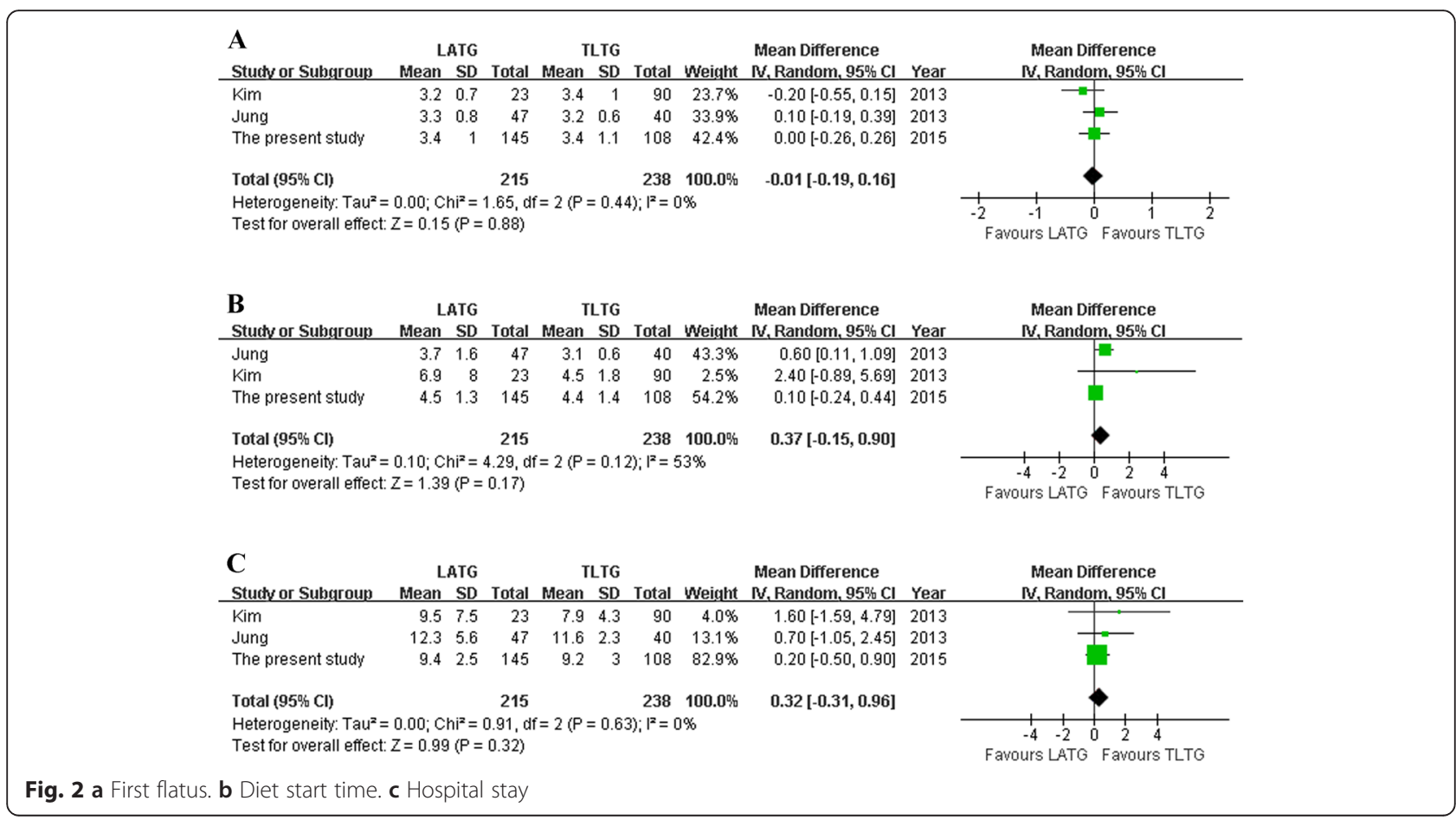



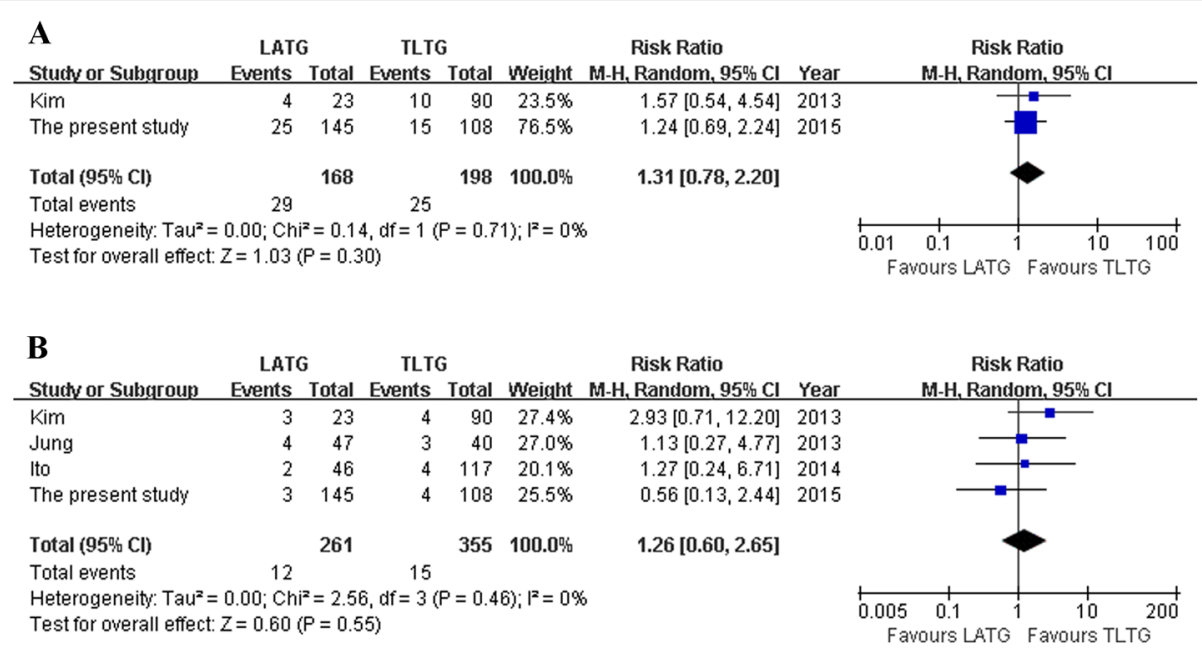

Fig. 3 a Overall complications. b Anastomosis-related complications

result in abdominal infection because of a contaminated OrVil tube and an overlapping anastomosis line on the esophagojejunostomy site. It is reported that TLTG with linear-stapled anastomosis is a simple, feasible choice with less postoperative reconstruction-related complications [18]. It has the additional limitation of requiring a sufficient esophageal length and necessitates taking down more of the esophagus within the mediastinum. In particular, when the more proximal esophagus needs to be transected, manipulation using an endoscopic linear stapler becomes extremely difficult. The traditional approach of circular stapler anvil is also limited for the placement of circular staplers which are improper in the laparoscopic surgery because of the absence of a matching tube and bigger size. The pneumoperitoneum was vulnerable to the placement, so the vision is unclear. The limitations resulted from the mechanical approach are overcome by hand-sewn esophagojejunostomy. The process of suture should be clearly noticed under high-definition laparoscopy, which makes anastomosis reliable. Besides, the operating space is large, and there is no tension in the whole anastomosis procedure. Also, this method does not need a longer esophageal stump. However, hand-sewn method requires the operators with rich experience in laparoscopic suture procedures, and it may take more time. Based on our experience, the linear stapler should be adjusted for patients with the lesions in the lower cardia and body as well as the fundus of the stomach. As for the patients with the lesions in the middle and upper cardia, the circular stapler can be selected to accommodate the

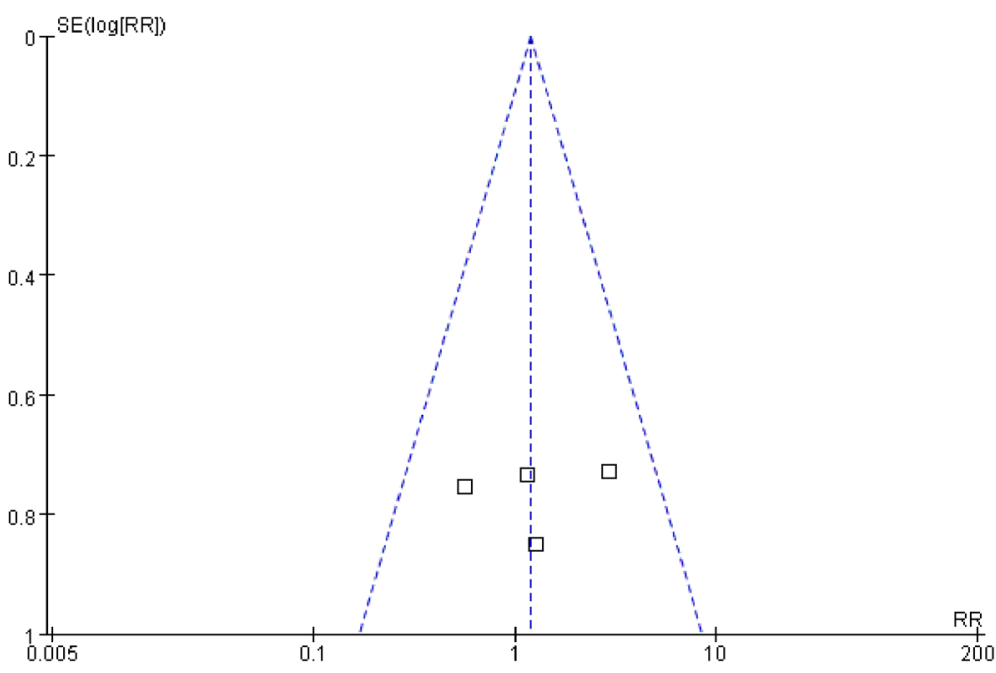

Fig. 4 Funnel plot of the anastomosis-related complications 
surgical margin. Finally, if the surgeon is experienced in laparoscopic hand-sewn technique, and it can be applied after total gastrectomy, no matter where the tumor is located.

There are several limitations to our studies. First, all of the outcomes resulted from East Asia, where the average BMI is lower than a common Western BMI. However, our results would be also suitable for Western patients, because intracorporeal reconstruction is easier than reconstruction through minilaparotomy in obese patients. Second, there is a difference in the time period when each of the surgical procedures was performed. LATG has been performed since March 2006, whereas TLTG has been performed since November 2007. Various operative factors related to the procedure itself, such as surgical instruments, sutures, and drugs, may have influenced the results. In addition, there may be differences in operator skill and perioperative care protocols among the surgical groups. Third, the majority of the studies analyzed focused only on total gastrectomy. However, the included studies had cases of proximal gastrectomy because the sample size of the remaining studies is too small for definitive conclusions and the larger the number of patients in a meta-analysis, the greater its power to detect a possible treatment effect [16]. Therefore, we did not exclude the study. Although, such a low number does not imply a significant bias, it still can lead to clinical heterogeneity.

\section{Conclusions}

The current study showed that TLTG is a feasible choice for gastric cancer patients, with comparable results to the LATG approach. However, more methodologically highquality comparative studies are required to adequately evaluate the status of TLTG.

\section{Abbreviations \\ CT: computed tomography; EUS: endoscopic ultrasonography; LAG: laparoscopic-assisted gastrectomy; LATG: laparoscopic-assisted total gastrectomy; LTG: laparoscopic total gastrectomy; MRI: magnetic resonance imaging; RR: risk ratio; TLDG: totally laparoscopic distal gastrectomy; TLG: totally laparoscopic gastrectomy; TLTG: totally laparoscopic total gastrectomy; WMD: weighted mean difference.}

\section{Competing interests}

The authors declare that they have no competing interests.

\section{Authors' contributions}

CK designed the study. CK, YJF, CDW, and WXF performed the operation. CJQ, WD, and YHM collected the literatures and conducted the analysis of pooled data. CK and PY wrote the manuscript. WXF proofread and revised the manuscript. All authors read and approved the final manuscript.

\section{Acknowledgements}

This work was supported by funds of the Department of Health of Zhejiang Province, China (grant no. 2015116990). The authors thank Hendi Maher from Australia for editing the English language.

Received: 28 September 2015 Accepted: 24 March 2016 Published online: 31 March 2016

\section{References}

1. World Health Organization, International Agency for Research on Cancer (2008) GLOBOCAN 2008: cancer incidence and mortality worldwide 2008. http://globocan.iarc.fr/. Accessed 18 March 2013

2. Kitano S, Shiraishi N, Uyama I, et al. A multicenter study on oncologic outcome of laparoscopic gastrectomy for early cancer in Japan. Ann Surg. 2007;245:68-72

3. Ikeda O, Sakaguchi Y, Aoki Y, Harimoto N, Taomoto J, Masuda T, Ohga T, Adachi , Toh, Okamura, Baba. Advantages of totally laparoscopic distal gastrectomy over laparoscopically assisted distal gastrectomy for gastric cancer. Surg Endosc. 2009;23:2374-9.

4. Guzman EA, Pigazzi A, Lee B, Soriano PA, Nelson RA, Benjamin Paz I, Trisal V, Kim J, Ellenhorn JD. Totally laparoscopic gastric resection with extended lymphadenectomy for gastric adenocarcinoma. Ann Surg Oncol. 2009;16:2218-23.

5. Song KY, Park CH, Kang HC, Kim JJ, Park SM, Jun KH, Chin HM, Hur H. Is totally laparoscopic gastrectomy less invasive than laparoscopy-assisted gastrectomy? Prospective, multicenter study. J Gastrointest Surg. 2008;12:1015-21.

6. Huscher CG, Mingoli A, Sgarzini G, Brachini G, Binda B, Di Paola M, Ponzano C. Totally laparoscopic total and subtotal gastrectomy with extended lymph node dissection for early and advanced gastric cancer: early and long-term results of a 100-patient series. Am J Surg. 2007;194:839-44. discussion 844.

7. Chen K, Xu X, Mou Y, Pan Y, Zhang R, Zhou Y, Wu, Huang C. Totally laparoscopic distal gastrectomy with $D_{2}$ lymphadenectomy and Billroth ॥ gastrojejunostomy for gastric cancer: short- and medium-term results of 139 consecutive cases from a single institution. Int J Med Sci. 2013;10:1462-70.

8. Chen K, Mou YP, Xu XW, Cai JQ, Wu D, Pan Y, Zhang RC. Short-term surgical and long-term survival outcomes after laparoscopic distal gastrectomy with $D_{2}$ lymphadenectomy for gastric cancer. BMC Gastroenterol. 2014;14:41.

9. Chen K, Mou YP, Xu XW, Pan Y, Zhou YC, Cai JQ, Huang CJ. Comparison of short-term surgical outcomes between totally laparoscopic and laparoscopic-assisted distal gastrectomy for gastric cancer: a 10-y single-center experience with meta-analysis. J Surg Res. 2015;194:367-74.

10. Cai J, Mou YP, Pan Y, Chen K, Xu XW, Zhou Y. Immunoglobulin G4associated cholangitis mimicking cholangiocarcinoma treated by laparoscopic choledochectomy with intracorporeal Roux-en-Y hepaticojejunostomy. World J Surg Oncol. 2014;12:363.

11. Cai JQ, Chen K, Mou YP, Pan Y, Xu XW, Zhou YC, Huang CJ. Laparoscopic versus open wedge resection for gastrointestinal stromal tumors of the stomach: a single-center 8-year retrospective cohort study of 156 patients with long-term follow-up. BMC Surg. 2015;15:58.

12. Pan Y, Mou YP, Chen K, Xu XW, Cai JQ, Wu D, Zhou YC. Three cases of laparoscopic total gastrectomy with intracorporeal esophagojejunostomy for gastric cancer in remnant stomach. World J Surg Oncol. 2014;12:342.

13. Yan JF, Xu XW, Jin WW, Huang CJ, Chen K, Zhang RC, Harsha A, Mou YP. Laparoscopic spleen-preserving distal pancreatectomy for pancreatic neoplasms: a retrospective study. World J Gastroenterol. 2014;20:13966-72.

14. Ajoodhea H, Zhang RC, Xu XW, Jin WW, Chen K, He YT, Mou YP: Intracorporeal esophagojejunostomy after totally laparoscopic total gastrectomy: a singlecenter 7-year experience. World J Gastroenterol 2016, 22 (online)

15. Kim HS, Kim MG, Kim BS, Lee IS, Lee S, Yook JH, Kim BS. Comparison of totally laparoscopic total gastrectomy and laparoscopic-assisted total gastrectomy methods for the surgical treatment of early gastric cancer near the gastroesophageal junction. J Laparoendosc Adv Surg Tech A. 2013;23:204-10.

16. Jung YJ, Kim DJ, Lee JH, Kim W. Safety of intracorporeal circular stapling esophagojejunostomy using trans-orally inserted anvil (OrVil) following laparoscopic total or proximal gastrectomy - comparison with extracorporeal anastomosis. World J Surg Oncol. 2013;11:209.

17. Ito H, Inoue H, Odaka N, Satodate H, Onimaru M, Ikeda H, Takayanagi D, Nakahara K, Kudo SE. Evaluation of the safety and efficacy of esophagojejunostomy after totally laparoscopic total gastrectomy using a trans-orally inserted anvil: a single-center comparative study. Surg Endosc. 2014:28:1929-35.

18. Kim HS, Kim BS, Lee S, Lee IS, Yook JH, Kim BS. Reconstruction of esophagojejunostomies using endoscopic linear staplers in totally laparoscopic total gastrectomy: report of 139 cases in a large-volume center. Surg Laparosc Endosc Percutan Tech. 2013;23:e209-16.

19. Takiguchi S, Sekimoto M, Fujiwara Y, Miyata H, Yasuda T, Doki Y, Yano M, Monden M. A simple technique for performing laparoscopic purse-string suturing during circular stapling anastomosis. Surg Today. 2005;35:896-9.

20. Usui S, Nagai K, Hiranuma S, Takiguchi N, Matsumoto A, Sanada K. Laparoscopy-assisted esophagoenteral anastomosis using endoscopic 
purse-string suture instrument "Endo-PSI (II)" and circular stapler. Gastric Cancer. 2008;11:233-7.

21. Omori T, Oyama T, Mizutani S, Tori M, Nakajima K, Akamatsu H, Nakahara M, Nishida T. A simple and safe technique for esophagojejunostomy using the hemidouble stapling technique in laparoscopy-assisted total gastrectomy. Am J Surg. 2009;197:e13-7.

22. Jeong O, Park YK. Intracorporeal circular stapling esophagojejunostomy using the transorally inserted anvil (OrVil) after laparoscopic total gastrectomy. Surg Endosc. 2009;23:2624-30.

23. Wang ZQ, Cai ZM, Chen J, Lei X, Luo HX, Yu PW. A modified method of laparoscopic side-to-side esophagojejunal anastomosis: report of 14 cases. Surg Endosc. 2008;22:2091-4.

Submit your next manuscript to BioMed Central and we will help you at every step:

- We accept pre-submission inquiries

- Our selector tool helps you to find the most relevant journal

- We provide round the clock customer support

- Convenient online submission

- Thorough peer review

- Inclusion in PubMed and all major indexing services

- Maximum visibility for your research

Submit your manuscript at www.biomedcentral.com/submit
Biomed Central 